\title{
Stellar Populations in M31 from Broad-band Photometry
}

\author{
Antti Tamm ${ }^{1}$, Elmo Tempel ${ }^{1,2}$, Peeter Tenjes $^{1,2}$ and Taavi Tuvikene ${ }^{1,3}$ \\ ${ }^{1}$ Tartu Observatory, Estonia \\ ${ }^{2}$ The University of Tartu, Estonia \\ ${ }^{3}$ Vrije Universiteit Brussel, Belgium, \\ e-mail: atamm@aai.ee
}

\begin{abstract}
Due to its proximity, size, complex structure and high inclination angle, M31 offers an excellent opportunity for studying galactic structures outside the Milky Way and for drawing implications for their cosmological origin. We have studied the stellar populations of M 31 using the Sloan Digital Sky Survey (SDSS) photometry and the Spitzer far-infrared (FIR) mappings of dust. Combining these data, we have constructed a 3-dimensional model of the galaxy, laying constraints on the intrinsic (dust-free) properties of the galaxy and its stellar populations: their apparent and intrinsic luminosities, luminosity distributions, colours, shapes and sizes. We have interpreted the derived spectral energy distributions with synthetic stellar populations created with the Starburst99 software, in order to constrain the ages and masses of the stellar components.
\end{abstract}

Keywords. galaxies: individual (M 31), galaxies: stellar content, galaxies: structure, galaxies: photometry, ISM: dust, extinction

Because of the short integration times required, broadband photometry is a favourable technique for studying the structure of nearby galaxies. For the same reason, estimating star formation histories and stellar masses on the basis of broad-band colour indices has become a common practice, especially in analyses of extensive galaxy samples and high-redshift objects. However, the actual uncertainties arising from the loss of spectral information and from the age-metallicity degeneracy often remain unknown. Thus it is interesting to conduct a corresponding analysis for galaxies in the local Universe, for which a wide variety of additional observational data can be collected at sufficient spatial resolution and depth. In such cases, ultraviolet through near-infrared photometry can be used for tracing the distribution and the chemical composition of the stellar content of galaxies. FIR observations can be used to map the interstellar dust and to take into account its effects on the photometry. And finally, stellar population synthesis models can be used to estimate stellar mass-to-light ratios, which in turn can be used to constrain the dark matter content of galaxies.

In this work we have tested the feasibility of broad-band photometry for studying the properties the stellar content of the nearby galaxy M31.

The photometry is based on probably the best optical imaging available for the whole of M31, the SDSS, in which this extended galaxy was observed through the ugriz filters on October 6th, 2002, resulting in a homogeneous dataset of 672 frames in 5 passbands, with the resolution of $0.396 \mathrm{arcsec} / \mathrm{px}$. A crucial step during image processing was the correct removal of the sky background, which turned out to contain several gradients and fluctuations. Foreground stars and background galaxies were detected and masked with the help of the SExtractor software (Bertin \& Arnouts 1996).

A model galaxy was fitted to the SDSS imaging, assuming the galaxy to consist of axially symmetric stellar components with constant colour indices. Looking for a balance 
between a simple model with minimal degeneracies and an adequate description of the observational data, we decided to distinguish the following stellar components: a bulge, an old disc, a young disc and a halo. The spatial luminosity density distribution of each component was approximated by the Einasto's law

$$
l(a)=l_{0} \exp \left[-\left(\frac{a}{k a_{0}}\right)^{1 / N}\right],
$$

where $l_{0}=h L /\left(4 \pi q a_{0}^{3}\right)$ is the central density and $L$ is the component luminosity; $a=\sqrt{r^{2}+z^{2} / q^{2}}$, where $r$ and $z$ are two cylindrical coordinates; $a_{0}$ is the harmonic mean radius. The coefficients $h$ and $k$ are normalising parameters, depending on $N$. The luminosity density distributions of individual components were summarised and projected along the line of sight according to the inclination angle of the galaxy, allowing a comparison to the observed surface brightness distribution.

In order to take the intrinsic dust effects into account, a dust disc was implanted in the model. To this end, far-infrared imaging of M 31 by the Spitzer Space Telescope was used to derive dust distribution, temperature and finally the extinction along each line of sight. In general, the model provided a good fit to the data, while some uncertainties remained in deriving the relation between the FIR flux and extinction. We estimated the total intrinsic extinction of M 31 in the $V$ passband to be $0.1 \mathrm{mag}$ and found the following intrinsic integral colour indices for M31: $(U-B)_{0}=0.35 \mathrm{mag} ;(B-V)_{0}=0.86 \mathrm{mag}$; $(V-R)_{0}=0.63 \mathrm{mag} ;(R-I)_{0}=0.53 \mathrm{mag}$; the total intrinsic absorption-corrected luminosities of M31 in the $B$ and the $V$ filters are 4.10 and 3.24 mag, respectively. More details about the photometry, dust properties, extinction calculations and the galactic model construction can be found in Tempel et al. (2000, 2011).

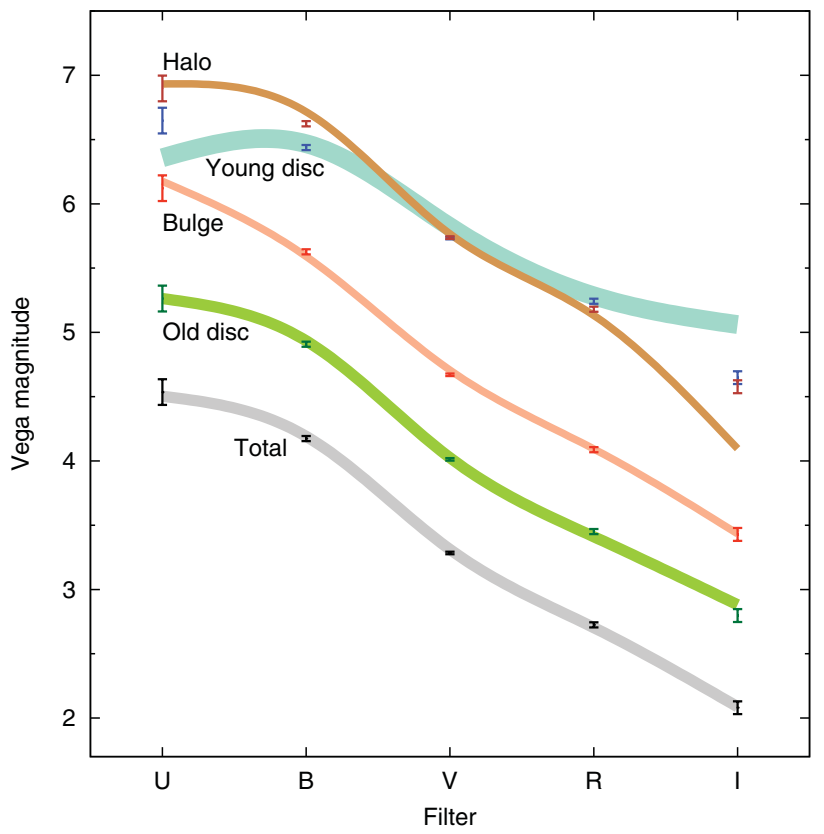

Figure 1. Extinction-corrected $U B V R I$ luminosities of M 31. Errorbars indicate the observed values together with uncertainties, as derived from the SDSS imaging. Solid lines represent the best-fit synthetic stellar populations, calculated with Starburst99. Line widths indicate the approximate uncertainties of the models. 
Table 1. Main properties of the stellar populations of M 31

\begin{tabular}{lcccc}
\hline Population & $\begin{array}{c}m_{V}^{*} \\
(\mathrm{mag})\end{array}$ & $\begin{array}{c}L_{V}^{*} \\
\left(10^{10} \mathrm{~L}_{\odot}\right)\end{array}$ & {$[\mathrm{M} / \mathrm{H}]^{* *}$} & $\begin{array}{c}M \\
\left(10^{10} \mathrm{M}_{\odot}\right)\end{array}$ \\
\hline Bulge & 4.71 & 0.70 & 0.0 & $2.8 \pm 0.5$ \\
Old disc & 4.02 & 1.25 & -0.3 & $3.6 \pm 0.6$ \\
Young disc & 5.82 & 0.24 & -0.3 & $0.10 \pm 0.07$ \\
Halo & 5.77 & 0.25 & -0.7 & $0.8 \pm 0.3$ \\
\hline Total & 3.31 & 2.42 & - & $7.3 \pm 1.1$ \\
\hline
\end{tabular}

Notes: ${ }^{*}$ Luminosities are corrected for intrinsic dust extinction.

** Metallicity values are combined from the literature. See text for details.

The photometric model yielded a five-bin spectral energy distribution for each of the stellar components, corresponding to the fluxes through the five SDSS filters. Since the intrinsic reddening had already been taken into account by the model, these distributions could be compared directly to the spectral energy distributions proposed by chemical evolution models for stellar populations of different ages and metallicities. We used the Starburst99 chemical evolution model (Leitherer, Schaerer, Goldader, et al. 1999) to conduct such a comparison for each of the modelled stellar component. In order to break the degeneracy between the effects of older ages and higher metallicities, estimates for the distribution of the metallicity were taken from the literature. We fixed the bulge metallicity at $[\mathrm{M} / \mathrm{H}]=0$ according to the measurements by Sarajedini \& Jablonka, (2005) and the disc metallicity at $[\mathrm{M} / \mathrm{H}]=-0.3$ according to Bellazzini, Cacciari, Federici, et al. (2003), Worthey, España, MacArthur, et al. (2005), and Brown, Smith, Ferguson, et al. (2006). In the outer regions, we relied on the results by Brown, Smith, Ferguson, et al. (2006) and Koch, Rich, Reitzel, et al. (2007), setting $[\mathrm{M} / \mathrm{H}]=-0.7$. Recent studies have recovered an additional diffuse and extended stellar population with still lower metallicities, possibly surrounding much of the local group (Ibata, Martin, Irwin, et al. 2007). However, we have neglected this component in our model because of its negligible contribution to the total luminosity of the galaxy and the limited size of the SDSS imaging.

Figure 1 indicates that the spectral energy distributions of the individual stellar components can be well matched with realistic synthetic populations. As expected, the bulge and the halo are best fitted with old (about 10-12 Gyr) stellar populations, while the disc can be approximated as a combination of a thick disc of old (about 8 Gyr) and a thin disc of young (about 1 Gyr) stellar populations. The mismatch between the model and observed fluxes of the young disc and halo in $U$ and $I$ filters is probably related to difficulties in the sky background determination for the Sloan $u$ and $z$ filters.

The chemical evolution model proposes also mass-to-light ratios for the stellar components. Considering the uncertainties of metallicity and age of the populations, we find the following mass-to-light ratios for the bulge, old disc, young disc and halo of M 31: $M / L_{V}=4.2 \pm 0.5 \mathrm{M}_{\odot} / \mathrm{L}_{\odot}, 2.9 \pm 0.3 \mathrm{M}_{\odot} / \mathrm{L}_{\odot}, 0.4 \pm 0.2 \mathrm{M}_{\odot} / \mathrm{L}_{\odot}$, and $3.2 \pm 0.6 \mathrm{M}_{\odot} / \mathrm{L}_{\odot}$, respectively. The total stellar mass of M 31 is thus $7.3 \pm 0.9 \times 10^{10} \mathrm{M}_{\odot}$.

We conclude from our analysis that broad-band photometry can be a useful tool for obtaining rough estimates for the ages and masses of stellar populations. The uncertainties can be considerably reduced if additional information about the intrinsic dust effects and stellar metallicities is available.

We acknowledge the financial support from the Estonian Science Foundation grants 7146, 7765, 7115 and the Estonian Ministry for Education and Science research project SF0060067s08. We are pleased to thank the SDSS Team for the publicly available data releases and the developers of Starburst99 for making the software conveniently handy. 


\section{References}

Bellazzini, M., Cacciari, C., Federici, L., Pecci, F. F., \& Rich, M., 2003, A\&AA, 405, 867

Bertin, E. \& Arnouts, S. 1996, A\&AS, 117, 393

Brown, T. M., Smith, E., Ferguson, H. C., Rich, R. M., Guhathakurta, P., Renzini, A., Sweigart, A. V., \& Kimble, R. A., 2006, ApJ, 652, 323

Ibata, R., Martin, N. F., Irwin M., Chapman, S., Ferguson, A. M. N., \& Lewis, G. F., McConnachie A. W., 2007, ApJ, 671, 1591

Koch, A.; Rich, R. M., Reitzel, D. B., Mori, M., Loh, Y.-S., Ibata, R., Martin, N., Chapman, S. C., Ostheimer, J., \& Majewski, S. R.; Grebel, E. K., 2007, AN, 328, 653

Leitherer, C., Schaerer, D., Goldader, J. D., González Delgado, R. M., Robert, C., Kune, D. F., de Mello, D. F., Devost, D., \& Heckman, T. M. 1999, ApJS, 123, 3

Sarajedini, A. \& Jablonka, P. 2005, AJ, 130, 1627

Tempel, E., Tamm, A., \& Tenjes, P. 2010, A\& A, 50991

Tempel, E., Tuvikene, T., Tamm, A., \& Tenjes, P. 2011, A\&A, 526, 155

Worthey, G., España, A., MacArthur, L. A., \& Courteau, S. 2005, ApJ, 631, 820 